\title{
Penis problems, treatment with VIPP cellsonic therapy
}

\author{
Andrew Hague* \\ Professor of Advanced Medicine, President, Cellsonic, Manufacturers of Medical Equipment, UK
}

\section{Introduction}

Doctors will boast that their patients are not shy and tell them everything but that is not how it is. Patients, especially men, are a hindrance to themselves. Erectile dysfunction or the more embarrassing bent knob of Peyronie's is something most men fear to admit. Even when they do summon the courage to talk to the doctor the best they can do is euphemise. This tells the doctor that the problem is more than physical, it is also psychological. Treat with care.

The patient will be referred to a urologist. The penis is unique. Nowhere else on the male is there anything similar and, in the female, there is no equivalent and yet the failure of this inflatable organ shares medical problems with other organs. Explain to the patient that the penis is a balloon that inflates with blood and he may begin to understand. If the blood cannot flow into the penis, it will not expand. The blockage can be similar to blockages that can occur elsewhere in the body and these are issue in which a vascular surgeon specialises. Angiogenesis is the formation of new blood vessels and that is the clue to the cure. What helps with heart disease can also be applied to the penis.

About twenty years ago when orthopaedic surgeons learned to heal broke bone with lithotripters derived from urology machines for breaking kidney stones, the discovery of wound healing was also made. This revealed that tissue formation was promoted by the machine's pressure pulses. Improved vascularisation encouraged fibroblasts which led soon after to finding further applications with damaged penises among them.

There are two problems for the penis. The first is the failure of blood to inflate the penis and this is called erectile dysfunction. The second problem is different because the blood pressure is adequate, but the tissues are damaged and cause the penis to erect bent. This is generally called Peyronie's disease after François Gigot de La Peyronie in France in 1743 described a disorder characterized by induration of the corpora cavernosa of the penis.

About twenty years ago, I attended a seminar on erectile problems organised by a patient through contacts made on the internet. This was a surprisingly sensible arrangement the likes of which I have not seen since. Four companies with machines to do the treatments attended and about twenty suffering men with their wives. In almost every case, it was the wife who had sought help and brought her husband along and that told me a lot about the different approaches by men and women on these subjects. What became apparent is that men are embarrassed, dislike medications and will only seek help if they can be anonymous as they can, sometimes, on the internet. The cause of Peyronie's disease is uncertain and cannot be blamed on diet, sexual activity be it too much or too little, exercise, smoking or anything else that could be called wrong.
There can be genetic causes of tissue tightening and the affliction in the penis is only one occurrence. The same patient may also have Dupuytren's contracture of the hand caused by the fascia rather than the tendons. Ledderhose disease is the tension of the plantar fascia of the feet. Frozen shoulder where the lining around the shoulder joint becomes inflamed and stiffens up was said to be linked to Dupuytren's by H. Schaer in 1936. Knuckle pads on fingers were described by Garrod to often occur along with Dupuytren's disease.

Initially, conditions like Frozen Shoulder, Dupuytren's, Ledderhose, Peyronie's and Garrod's Pads were all viewed as separate diseases. Now all of these conditions are seen to have the same underlying cause, often called Dupuytren's diathesis or disposition and can be cured with CellSonic VIP (Figure 1).

The cure for erectile dysfunction and the bend of Peyronie's is the same by treating with a CellSonic VIPP (very intense pressure pulses) machine. No drugs are used so there are no side effects and it is nonsurgical. After being cured by CellSonic, the patient will be no more likely to get a recurrence of the disease than another patient who has never had it.

Prior to the use of CellSonic, the only remedy for Peyronie's was surgery. Cuts were made on the convex side of the bend to straighten the penis with the overall length reduced. That shame combined with the agony of surgery scared most men away. The true amount of suffering is unknown because men are too embarrassed to talk about it and even if they are and are told that they must have surgery they will then stop talking for sure and suffer in silence. That pitiful situation can now end. All it takes is ten minutes CellSonic treatment.

The CellSonic VIPP machine generates sudden bursts of sound that are pressure pulses to break a calcification or plaque. It also tells the body's immune system to repair damaged veins and arteries with angiogenesis to grow new blood vessels. All this is done without drugs or anaesthetic so there are no side effects.

The patient knows his penis and will have a good idea where the problem is. If it is in one place, the protocol is to aim 300 pulses at that place at energy level one, the lowest setting, with a shock head focussed at $5 \mathrm{~mm}$, especially designed for this purpose. If there are other areas in the penis where the blockages may be, they too can be given pulses to a maximum of 1500 over all the penis at one time. The pulses are $4 \mathrm{a}$ second so it only takes a few minutes.

${ }^{\star}$ Correspondence to: Andrew Hague, Professor of Advanced Medicine, President, Cellsonic, Manufacturers of Medical Equipment, UK, Tel: +1 315210 6307; E-mail: cellsonic.beauty@gmail.com

Received: December 04, 2018; Accepted: December 11, 2018; Published: December 13, 2018 


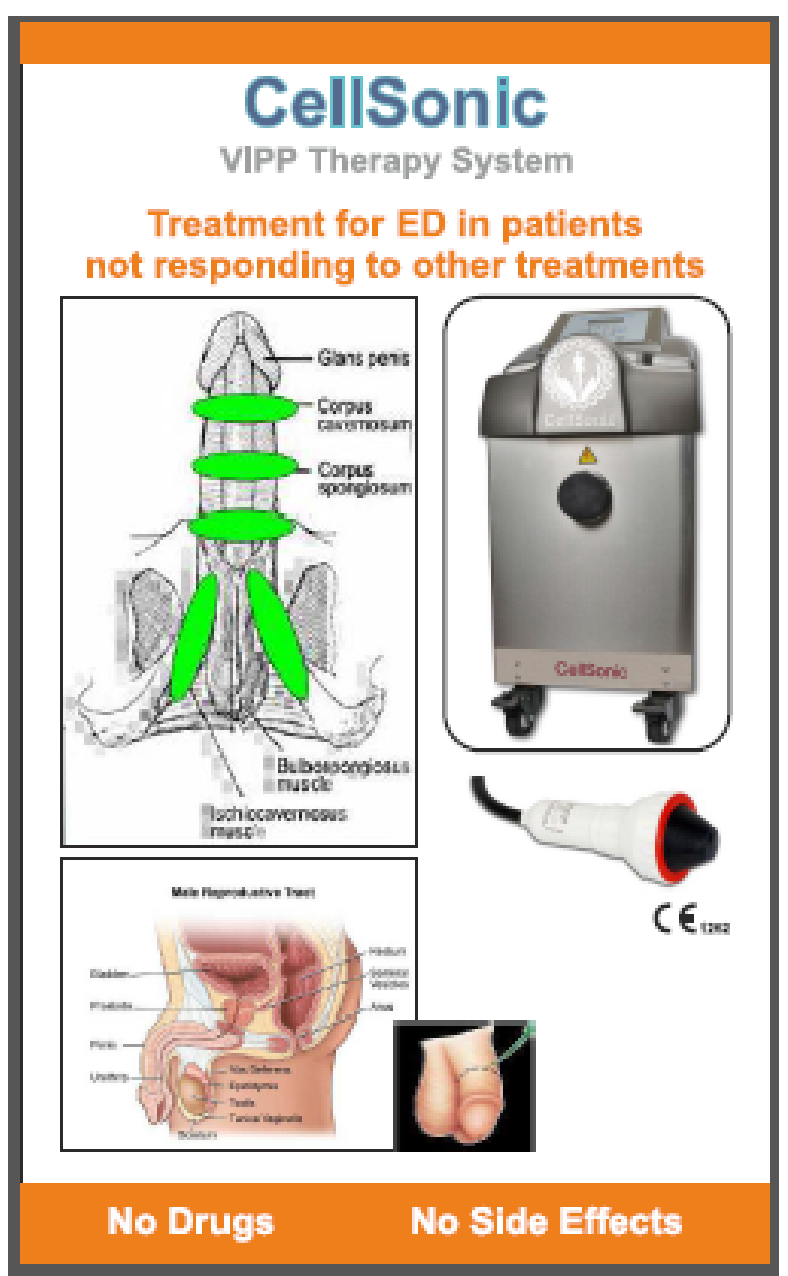

Figure 1. Treatment from Cellsonic VIPP therapy system
The easiest way to perform the operation is for the patient to stand and do the treatment himself. Let him feel what a VIPP pulse is by hitting his finger with one or two pulses and it will hurt so he knows this is a capable physical effect. The only nerves in the penis are on the end. Along the shaft of the penis are no nerves so no pain will be felt. Let the patient hold the shock head in one hand and his penis in the other. The doctor will supervise. Apply ultrasound gel between the penis and the membrane of the shock head so that the acoustic pulses are conducted through into the penis. After the first few pulses it will be obvious how easy and painless is the treatment. The machine can be set to stop after 300 shocks so there is no risk of doing too many. Let the pulses hit the suspected area from all sides; keep the shock head moving, not fixed on one spot. We have found that the patient will prefer this way of doing the treatment. Be sure to also aim at the root of the penis where it adjoins the body.

The treatment should be repeated once a week for nine weeks. New cells will be growing and continue to do so for up to a year after treatment, so the condition will continually improve. No drugs are required. Exercise a lot, never smoke, try not to drink alcohol and never take narcotic drugs.

\section{Protocol:}

$\begin{array}{ll}\text { Energy level } & 1 \\ \text { Shock head } & 5\end{array}$

$5 \mathrm{~mm}$ (the infinity head has also been used successfully)

Number of shocks $\quad 300$ to each site, maximum 1500 on all the penis in one treatment.

Number of treatments 9 at weekly intervals

Copyright: @2018 Hague A. This is an open-access article distributed under the terms of the Creative Commons Attribution License, which permits unrestricted use, distribution, and reproduction in any medium, provided the original author and source are credited. 